\title{
Tratamiento quirúrgico de la periimplantitis mediante colgajo de Widman modificado: detoxificación de la superficie implantaria Vs modificación de la superficie
}

\author{
LORENZO VIGNAU R* \\ SANZ CASADO JV** \\ MARTÍNEZ CORRIA R*** \\ BASCONES MARTÍNEZ A****
}

\begin{abstract}
Lorenzo Vignau R, Sanz Casado JV, Martínez Corria R, Bascones Martínez A. Tratamiento quirúrgico de la periimplantitis mediante colgajo de Widman modificado: detoxificación de la superficie implantaria Vs modificación de la superficie. Av Periodon Implantol. 2006; 18, 2: 75-82.
\end{abstract}

\begin{abstract}
RESUMEN
El fracaso tardío de los implantes hace plantearnos nuevas estrategias terapéuticas para resolver la perdida de hueso alrededor de los implantes a largo plazo (periimplantitis). Se presenta una técnica quirúrgica que preserve los tejidos marginales (colgajo de Widman Modificado) y comparar la estabilidad de los tejidos periimplantarios a 6 meses en una muestra de dos pacientes tratados uno de ellos con modificación de la rugosidad de la superficie: implantoplastia (test) y el otro con detoxificación de la superficie con clorhexidina (control). Material y Métodos: 2 mujeres que presentan signos clínicos de inflamación, supuración, sangrado al sondaje, profundidad de sondaje $>6 \mathrm{~mm}$ y evidencia de pérdida ósea radiográfica. Resultados: En ambos casos se produjo una disminución de los signos clínicos de inflamación a los 3 y 6 meses y una reducción de la profundidad de sondaje. Conclusión: el abordaje quirúrgico de la periimplantitis mediante el colgajo de Widman modificado es un abordaje efectivo para el tratamiento de defectos horizontales alrededor de los implantes con un mínimo trauma en los tejidos manteniendo la máximo la estética gingival.
\end{abstract}

\section{PALABRAS CLAVE}

Periimplantitis. Recesión.

Aceptado para publicación: Octubre 2005.

\section{INTRODUCCIÓN}

En la actualidad, el éxito o fracaso de la rehabilitación con implantes a corto plazo no es un tema a debate debido a la gran tasa de éxito y de supervivencia que las nuevas superficies han aportado a la implantología (1). Sin embargo el fracaso tardío de los implantes hace plantearnos nuevas estrategias terapéuticas para resolver la perdida de hueso alrededor de los implantes a largo plazo (periimplantitis) y para resolver las

\footnotetext{
* Odontólogo. Alomno Master de Periodoncia. Facultad de Odontología. Universidad Complutense de Madrid.

* $\quad$ Profesor Titular de Anatomía y Director del Instituto de Embriología de la Facultad de Medicina. Universidad Complutense de Madrid.

*** Médico-Estomatólogo. Cirujano Maxilofacial. Director de Bramermark Oseointegration Center. Madrid.

**** Catedrático de Medicina Bucal y Periodoncia. Departamento de Medicina y Cirugía Bucofacial (Estomatología III). Facultad de Odontología. Universidad Complutense de Madrid. España.
} 
secuelas que el tratamiento quirúrgico de la periimplantitis puede dejar al provocar una retracción de los tejidos periimplantarios.

Se denomina enfermedad periimplantaria a los cambios de tipo inflamatorio de los tejidos que rodean a un implante sometido a carga (2). Existe una relación causa-efecto entre la acumulación de placa bacteriana y el desarrollo de cambios inflamatorios de los tejidos blandos que rodean a los implantes que si no es eliminada se llegara al fracaso tardío de los implantes (5) y una serie de factores, como el estado médico, el tabaco, factores relacionados con el huésped, con el operador y con el tipo de implante que están asociados a un mayor riesgo de fracaso tardío y que complican el pronóstico a largo plazo de los implantes (7). Existe por tanto suficiente evidencia científica para demostrar una relación directa entre la microbiota oral y la presencia de periimplantitis y mucositis (10).

La Prevalencia de la periimplantitis ha sido observada en un rango de $1-19 \%$ de los casos (4).

Los signos y síntomas de la periimplantitis son:

Sangrado al sondaje, supuración, aumento de la profundidad de sondaje, dolor a la masticación o percusión, perdida ósea radiográfica y en casos avanzados movilidad progresiva del implante (6-9). Los patógenos más relacionados con la perdida ósea progresiva alrededor de los implantes presentan dos perfiles distintos:

- Bacterias asociadas con enfermedad periodontal gramnegativo como: Actinobacillus actinomycemccomitans, Porphyromonas gingivalis, Prevottella intermedia y espiroquetas (8).

- Bacterias no asociadas con de forma primaria periodontitis como: Staphylococcus spp Candida spp y bacilos entéricos (7).

El objetivo primario del tratamiento de la periimplantitis es el cese de la destrucción ósea para que este continúe función con tejidos periodontales sanos y secundariamente la regeneración de los tejidos perdidos siempre que sea posible (3).

Es muy importante comprender que las superficies a tratar en los casos de enfermedades periimplantarias son completamente distintas de las superficies radiculares naturales y que por tanto la eliminación de los depósitos bacterianos de la superficie de los implantes no siempre es fácil debido a la presencia de espiras y de la rugosidad que presentan las superficies dentarias (11).
En el caso de que la pérdida ósea sea avanzada el tratamiento quirúrgico será el tratamiento de elección para la descontaminación de la superficie de los implantes (6). En caso de que la perdida sea horizontal y los defectos del tamaño sean moderados, es decir, menores de $3 \mathrm{~mm}$, (20) la técnica quirúrgica de elección será el colgajo de reposición apical y técnicas resectivas de forma que se descontaminen de forma efectiva la superficie de los implantes se reduzca la profundidad de sondaje y se facilite al paciente una correcta higiene de estas superficies para mantener en salud los tejidos periimplantarios (12).

Un importante problema que nos podemos encontrar a la hora de tratar quirúrgicamente este tipo de entidad patológica es la exposición los implantes al reponer los tejidos apicalmente produciéndose un compromiso estético que sería máximo a nivel anterior que se acentuaría en casos de línea de sonrisa alta.

El objetivo de este estudio es describir una técnica quirúrgica que preserve los tejidos marginales (colgajo de Widman Modificado) y comparar la estabilidad de los tejidos periimplantarios a 6 meses en una muestra de dos pacientes tratados uno de ellos con modificación de la rugosidad de la superficie: implantoplastia (test) y el otro con detoxificación de la superficie con clorhexidina (control).

\section{MATERIAL Y MÉTODOS}

\section{PACIENTES}

Dos mujeres de 51 y 59 años de edad presentan signos clínicos de inflamación, supuración, sangrado al sondaje, profundidad de sondaje $>6 \mathrm{~mm}$ y evidencia de pérdida ósea radiográfica.

A ambos pacientes se les sometió antes de la cirugía a una profilaxis profesional y se les dio instrucciones de higiene oral. En la zona de los implantes afectados recibieron un raspado con curetas de teflón un mes antes de la cirugía. Al mes se reevaluó a las dos pacientes y se observó que seguían teniendo signos de inflamación.

\section{Paciente 1}

Mujer de 51 años, no fumadora, sin enfermedades sistémicas. Portadora de implantes Brånemark desde hace un año y medio. 


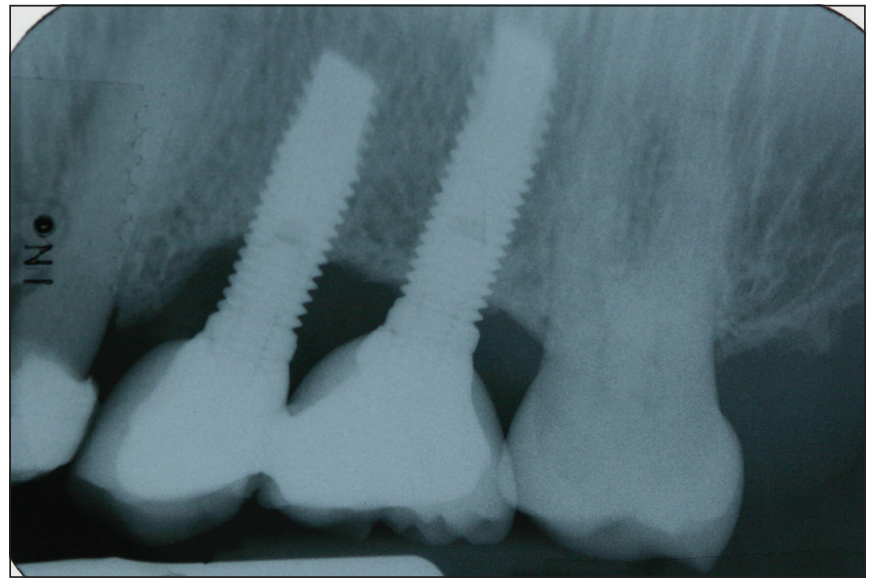

Fig. 1: Rx de paciente 1 .

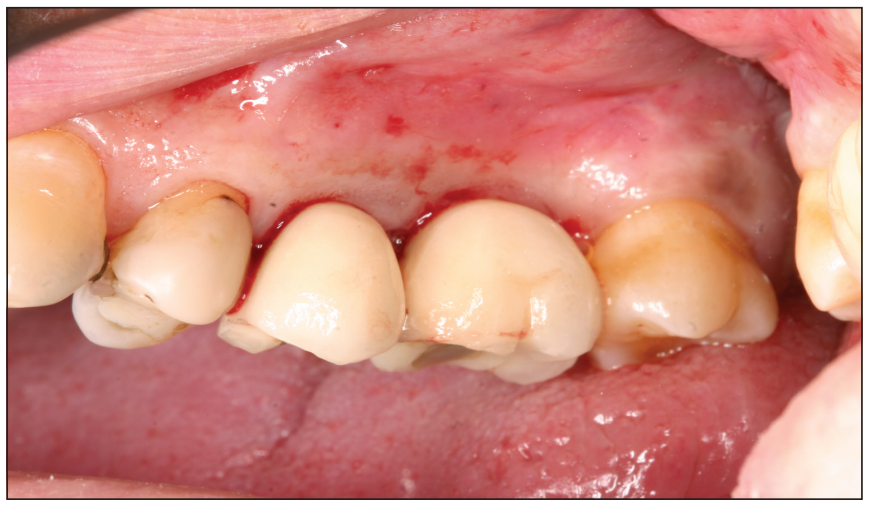

Fig. 2: Vista inicial de paciente 1.

\section{Paciente 2}

Mujer de 59 años, exfumadora, sin enfermedades sistémicas. Portadora de implantes ITI desde hace cuatro años.

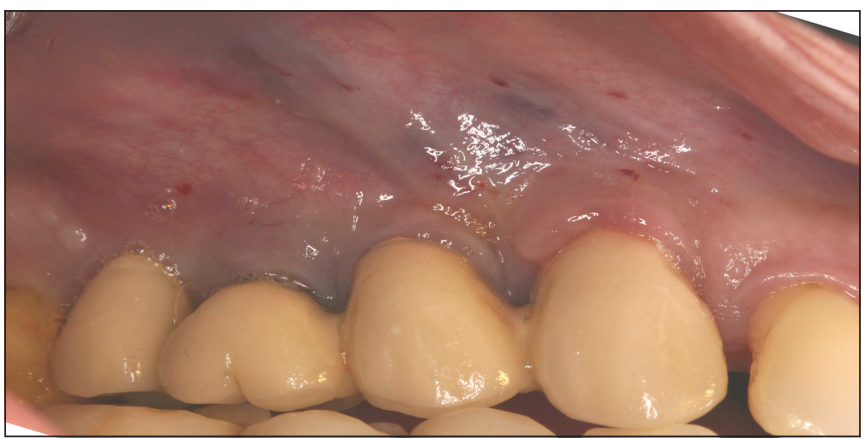

Fig. 3: Fotografía inicial de paciente 2.

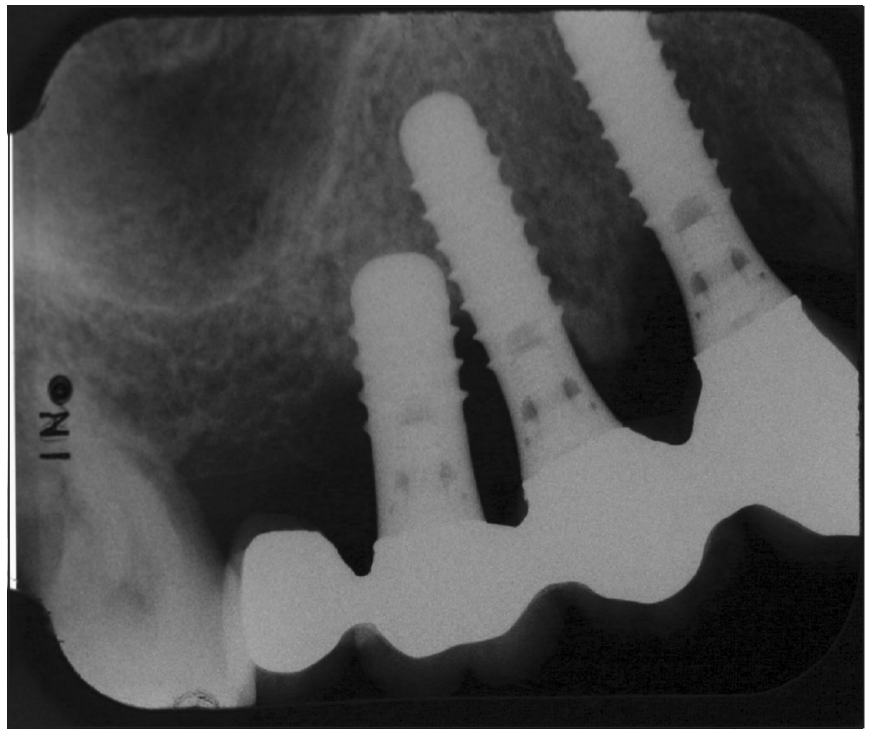

Fig. 4: $R x$ de paciente 2 .

\section{PROCEDIMIENTO QUIRÚRGICO}

Se llevó a cabo en ambos casos el mismo procedimiento:

Se infiltró Articaina (Ultracaine $®$ ). Con un bisturí n ${ }^{\circ} 15$ se realizó un colgajo de Widman modificado (13) llevado a cabo como de la siguiente manera:

Se realizó una primera incisión a medio mm de la margen gingival paralela al eje axial del diente. Una segunda incisión intrasulcular y una tercera incisión horizontal que conecta la primera incisión con la segunda por vestibular y en el extremo libre por mesial se realizó una incisión vertical liberadora que sobrepasa la

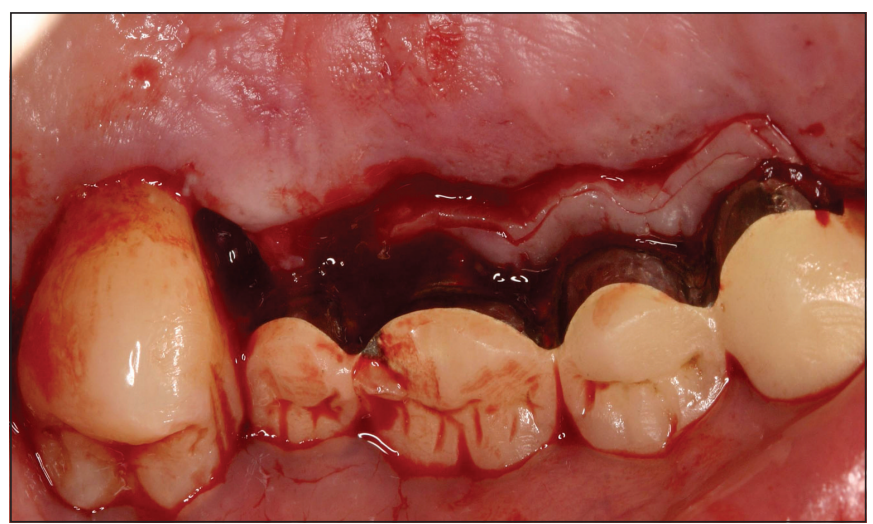

Fig. 5: Diseño de la incisión inicial por palatino de paciente 2. 
línea mucogingival para obtener un campo quirúrgico amplio.

En la cara palatina se diseñaron unos rodetes en función de la profundidad de sondaje a bisel interno adelgazando el espesor del paladar para conseguir una máxima adaptación de los tejidos (colgajo biselado).

Se levantó un colgajo a espesor total tanto en vestibular como por palatino y se eliminó el collar de tejido marginal incluyendo tanto el epitelio de la bolsa como el tejido inflamatorio de granulación. A continuación se eliminaron las aristas de hueso y se redujeron los defectos óseos con una fresa redonda de tungsteno bajo irrigación abundante.

\section{TRATAMIENTO DE LA SUPERFICIE DE LOS IMPLANTES}

\section{Paciente 1}

Después de la cirugía ósea se procedió a descontaminar las espiras expuestas mediante la irrigación una solución salina y después se aplicó durante tres minutos un gel de clorhexidina $0,2 \%$ durante tres minutos. Finalizado el proceso se suturó.

\section{Paciente 2}

En este caso se procedió a eliminar las espiras expuestas mediante turbina con una fresa redonda de tungsteno y se pulió la superficie con contraángulo de micromotor y fresas de goma. Finalmente se suturó.

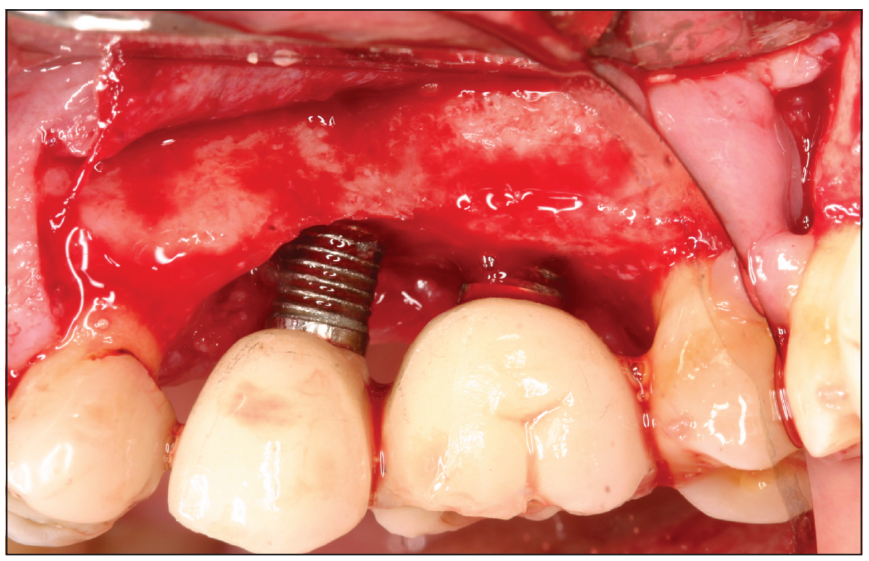

Fig. 6: Imagen del colgajo muco-perióstico de paciente 1.

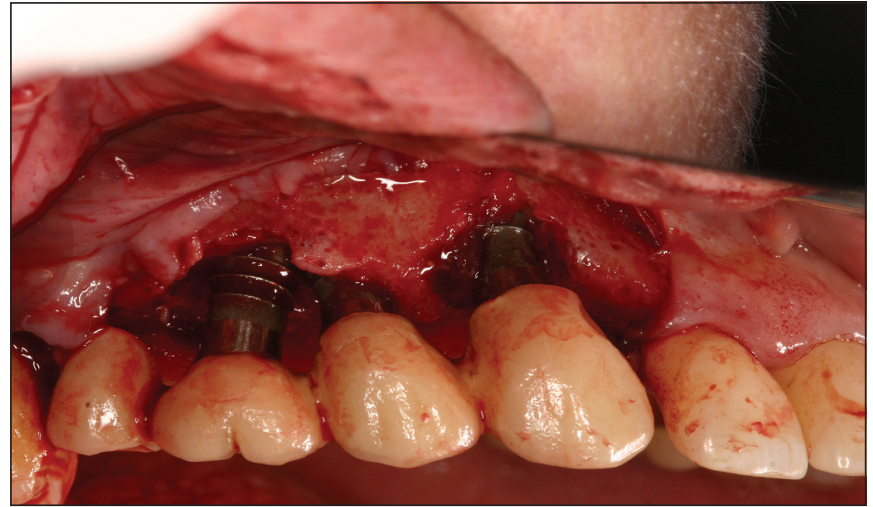

Fig. 7: Imagen del colgajo muco-perióstico de paciente 2.

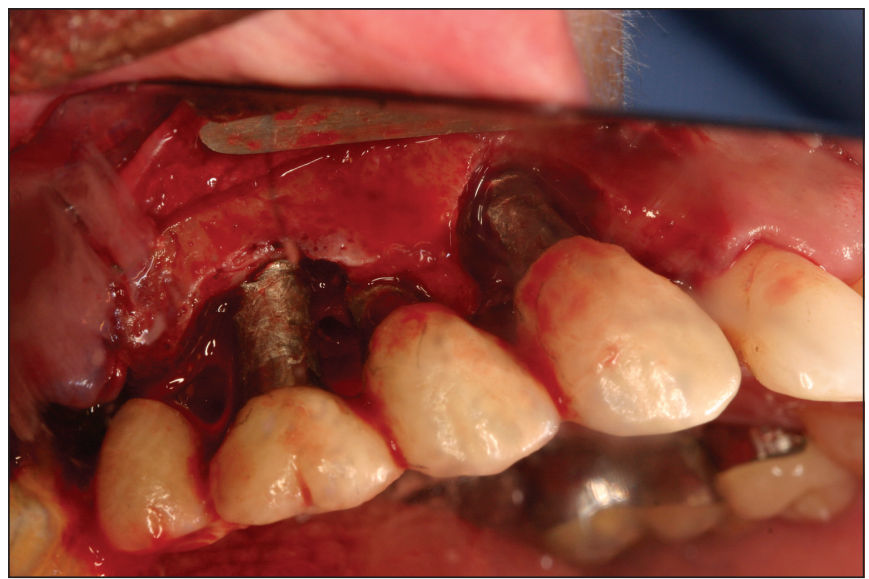

Fig. 8: Implantoplastia de paciente 2.

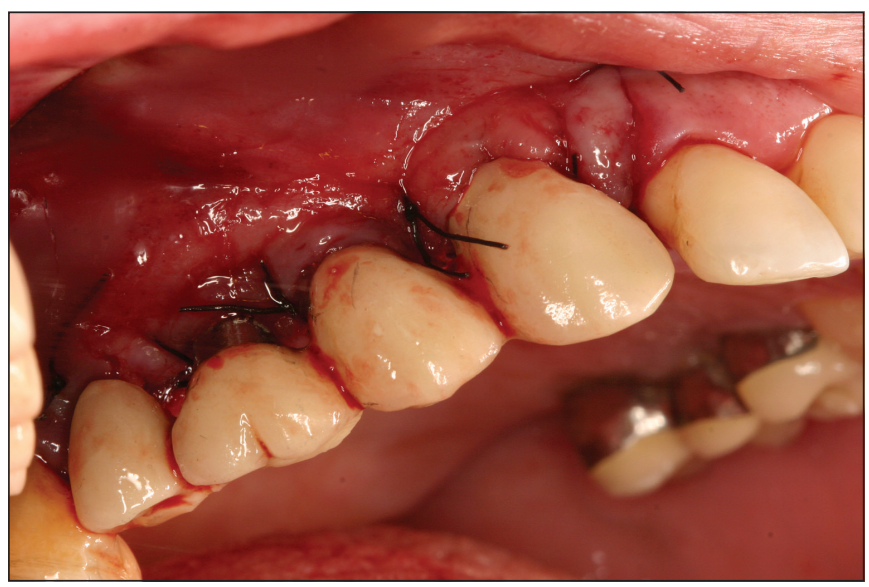

Fig. 9: Imagen de la sutura de paciente 2.

En ambos casos se administró enjuagues de clorhexidina $0,12 \%$ tres veces al día durante una semana. Una semana después de la cirugía se procedió a la retira- 


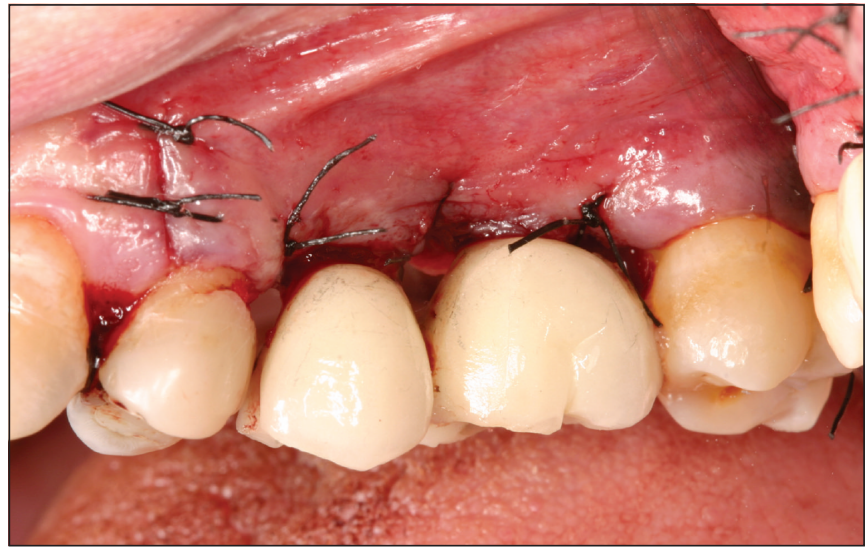

Fig. 10: Imagen de la sutura de paciente 1.

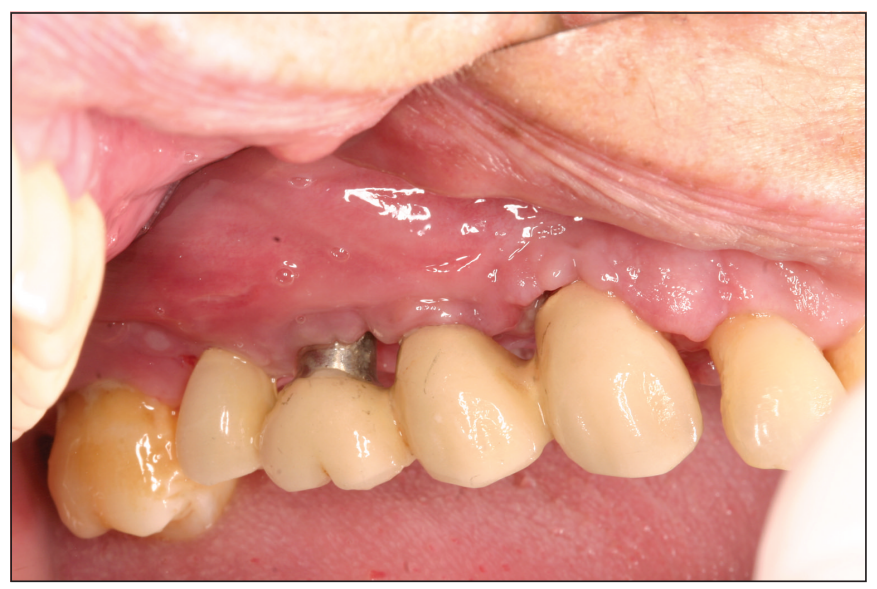

Fig. 11: Cicatrización a la semana del paciente 2.



Fig. 12: Cicatrización a la semana del paciente 1.

da de la sutura observándose una cicatrización correcta de los tejidos periimplantarios en ambos casos.

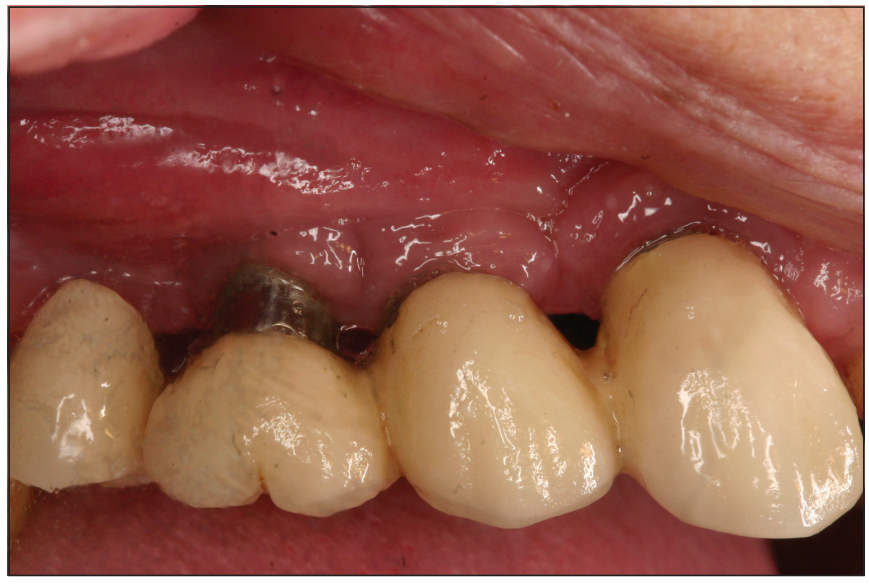

Fig. 13: Cicatrización a 6 meses del paciente 2.

Se observó una retracción ligera del margen gingival mas acentuada en el caso test debido a la situación mas apical del margen gingival.

\section{RESULTADOS}

En ambos casos se produjo una disminución de los signos clínicos de inflamación a los tres y 6 meses. Se produjo una reducción de la profundidad de sondaje que de 3-4 mm, de tal forma que no hubo ninguna localización con sondajes superiores a tres $\mathrm{mm}$ en ninguno de los dos casos tanto al mes como a los tres y seis meses.

Con respecto al sangrado no hubo sangrado al sondaje en ninguna localización a los tres y a los 6 meses al

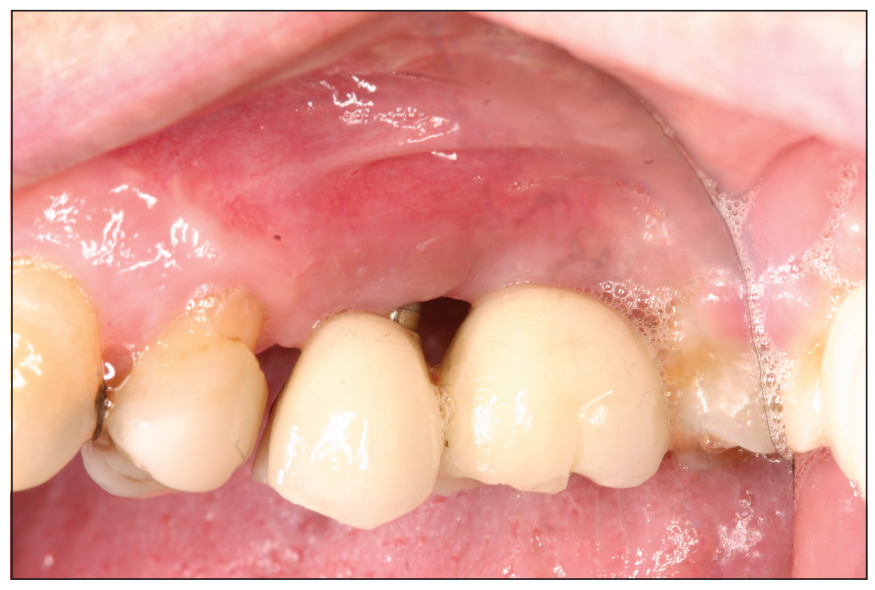

Fig. 14: Cicatrización a 6 meses del paciente 1 . 
igual que ocurrió con la supuración que también en desapareció tanto en el paciente test como en el control.

No se encontró ninguna diferencia clínica entre ninguno de los dos casos, produciéndose una mejoría significativa de los parámetros clínicos en ambos casos.

Con respecto a la recesión de los tejidos blandos, en ambos casos se produjo una ligera retracción del margen gingival que quedó estable a los tres meses y no se modificó durante los siguientes seis meses de observación. Se produjo una mayor recesión en el caso test debido a una mayor perdida ósea inicial en el implante mas posterior sobre todo a nivel interproximal como se observa en la imagen. De todos modos ninguna de las dos pacientes refirió quejas en relación con la estética.

\section{DISCUSIÓN}

El enfoque terapéutico de la periimplantitis con defectos supraóseos alrededor de la superficie de los implantes diferentes aspectos (21):

- Eliminar la placa bacteriana supragingival y acceder quirúrgicamente levantando un colgajo para poder eliminar el tejido de granulación y exponer la superficie de los implantes de tal forma que se pueda detoxificar la superficie de los implantes, modificando la rugosidad de la superficie de los implantes.

- Una vez se ha limpiado por completo el área afectada en caso de ser necesario de debe corregir la arquitectura del hueso para obtener una anatomía fisiológica. Como punto final se debe establecer un correcto control de placa (2)

Para conseguir estos objetivos las distintas opciones terapéuticas que disponemos son los diferentes abordajes quirúrgicos (6):

1. Desbridamiento cerrado

2. Desbridamiento abierto

3. Injertos óseos y sustitutos óseos

4. Membranas

5. Injertos + membranas

Siguiendo con el tratamiento clásico de la enfermedad periodontal, una vez diagnosticada la patología se debe de proceder al raspado y alisado radicular previo a la cirugía ya sea resectiva o regenerativa.
Existe diversas alternativas a la hora de tratar mecánicamente la superficie de los implantes, las unidades de polvo abrasivo aumentan la rugosidad de la superficie del implante y se retienen parte de estas partículas, la aplicación de agua + polvos a presión pueden provocar complicaciones, enfisemas así como destrucción de hueso observado radiográficamente alrededor de los implantes (16).

En estos dos casos que presentados, antes del tratamiento quirúrgico se procedió a la limpieza de los implantes mediante curetas de teflón. Este tipo de instrumento no modifica de ningún modo la superficie de los implantes y por tanto no raya la superficie del implante (18). Existen estudios que aunque demuestran el beneficio del uso de las curetas de teflón (19), aunque en estos dos casos clínicos presentados el hecho de utilizar este tipo de instrumentos, no produjo prácticamente ninguna mejoría de los parámetros clínicos y por tanto se pasó al abordaje quirúrgico de la enfermedad.

Con respecto al tratamiento quirúrgico mas efectivo para tratar este tipo de defectos existen diferencia en la literatura revisada sobre cual es la terapia mas adecuada y esta variará en función del tipo de defecto y en función del tipo de detoxificación de la superficie de los implantes.

Según algunos estudios el abordaje mas indicado para el tratamiento de los implantes con perdida de hueso en sentido horizontal parece ser el desbridamiento abierto (12).

En el caso de que la perdida de hueso sea en sentido horizontal el abordaje quirúrgico mas indicado será el desbridamiento abierto debido a la escasa eficacia de la terapia antibiótica/antimicrobiana sin desbridamiento de las lesiones y detoxificación de la superficie del implante. (3)

En caso de defectos verticales, la terapia regenerativa parece aportar un beneficio adicional sobre la resectiva debido a que incluso se a llegado a conseguir la reosteointegración completa de la superficie expuesta (3).

Se necesitan estudios a largo plazo en humanos para determinar el abordaje más adecuado para el tratamiento de esta enfermedad.

Con respecto al modo de tratar la superficie de los implantes existen autores que proponen la simple limpieza de las superficies, propugnando que en implantes con superficie rugosa, se debe utilizar el método 
de detoxificación mas simple (suero salino + CLX) (14) sobre todo cuando se combina con injerto autólogo particulado + PTFEe (3)

Otros consideran sin embargo positivo el hecho de modificar la topografía de la fijación implantosoportadas (implantoplastia) de tal forma que la cirugía resectiva + implantoplastia, parece influir de manera positiva en la supervivencia de los implantes afectados por periimplantitis y en parámetros clínicos como profundidad de sondaje, supuración y sangrado (12)

En este articulo se presentan dos casos de periimplantitis uno de ellos tratado con suero salino + clorhexidina y otro tratado con modificación de la topografía de los implantes. En ambos casos el resultado a seis meses fue exitoso y que se produjo una disminución de los signos clínicos de inflamación, por tanto no se puede concluir cual de los dos enfoques es más efectivo que el otro. Lo que si se ha observado es la mejoría evidente que el tratamiento quirúrgico de este tipo de defectos proporciona a los casos de periimplantitis al compararlos con el desbridamiento cerrado con curetas de teflón.

Una consideración muy importante que no aparece en los distintos estudios revisados es la secuela estética que el abordaje quirúrgico de esta patología puede producir debido a la exposición de los implantes (17). Hay estudios proponen el colgajo de reposición apical como técnica de elección para el tratamiento de las periimplantitis (12), pero no tienen en cuenta el efecto que este produce al ubicar el margen gingival a un nivel mas apical mostrando el color metálico del titanio.

En este artículo se propone el abordaje quirúrgico del colgajo de Widman modificado como técnica de elección del tratamiento de la periimplantitis debido a su efectividad a la hora de tratar este tipo de defectos, a su estabilidad a 6 meses y al mantenimiento de la estética debido al mantenimiento de la inserción por posición coronal del margen gingival inherentes a este colgajo.

Se presentan dos pacientes que presentan un a mínima recesión de los tejidos blandos tras el tratamiento con Widman modificado. Esta recesión se presenta a la semana y es estable a los 6 meses.

Por tanto se puede concluir que el abordaje quirúrgico de la periimplantitis mediante el colgajo de Widman modificado es un abordaje efectivo para el tratamien- to de defectos horizontales alrededor de los implantes con un mínimo trauma en los tejidos manteniendo la máximo la estética gingival.

De todos modos, los estudios existentes son estudios muy poco controlados y, por tanto, poco favorables para obtener conclusiones claras.

Se necesitan estudios a largo plazo en humanos para determinar el abordaje más adecuado para el tratamiento de esta enfermedad.

\section{ABSTRACT}

The late failiure of the implants make us design new therapeutic strategies for solving the bone lost around the implants in long term (periimplantitis). It is presented a surgical approach that preserve the marginal tissues (modified Widman flap) in two pacients one of them treated with a modification of the implant roughtness: implantoplasty (test) and other case with detoxification of the surface with clorhexidine (control). Material and Methods: 2 Women that present clinical signs of inflamation, supuration, bleeding on probing, probing depth $>6 \mathrm{~mm}$ and evidece of radiographic bone lost. Results: in both cases the was a reduction of the inflamatories clinical signs a 3 and 6 months and a reduction of the probing depth. Concliusion: The surgical approach with modified Widman flap it is an effective approach for the treatment of the horizontal defects around the implants with a minimal trauma mantaining the gingival aesthetic.

\section{KEY WORDS}

Periimplantitis. Recession.

\section{BIBLIOGRAFÍA}

1. Van Steenberghe D, Quirynen M. \& Naert I. (1999b) Survival and success rates with oral endosseous implants. In: Lang, N.P., Karring, T., Lindhe, J., eds. Proceedings of the 3rd European Workshop on Periodontology. Berlin: Quintessence Books, 242-54.

2. Mombelli A, Lang NP. The diagnosis and treatment of periimplantitis. Periodontol 2000 1998;17:63-76.

3. Schou S, Holmstrup P, Jorgensen T, Stoltze K, HjortingHansen E, Wenzel A. Autogenous bone graft and ePTFE 
membrane in the treatment of peri-implantitis. I. Clinical and radiographic observations in cynomolgus monkeys. Clin Oral Implants Res. 2003 Aug; 14(4):391-403.

4. Van Steenberghe D, Lekholm U, Bolender C, Folmer T, Henry P, Herrmann I, Higuchi K, Laney W, Linde'n, U. \& A ${ }^{\circ}$ strand, P. (1990) The applicability ofsseointegrated oral implants in the rehabilitation of partial edentulism: a prospective multicenter study on 558 fixtures. International Journal of Maxillofacial Implants 5:272-81.

5. Pontoriero R, Tonelli MP, Carnevale G, Mombelli A, Nyman SR, Lang NP. Experimentally induced periimplant mucositis. Clin Oral Implants Res 1994:5:254-9.

6. Sánchez-Garcés $\mathrm{M}^{\mathrm{a} A}$, Gay-Escoda C. Periimplantitis. Med Oral Patol Oral Cir Bucal 2004;9 Suppl:S63-74.

7. Leonhardt A, Renvert S, Dahlen G. Microbial findings at failing implants. Clin Oral Implants Res. 1999 Oct;10(5): 339-45.

8. Heydenrijk K, Meijer HJA, Van der Reijden WA, Raghoebar GM,Vissink A, Stegenga B. Microbiota around root-form endosseous implants: a review of the literature. Int J Oral Maxillofac Implants 2002;1 7:829-38.

9. Quirynen, M., De Soete, M. \& van Steenberghe, D Infectious risks for oral implants: a review of the literature. Clinical Oral Implants Research. (2002)13:1-19.

10. Lang NP, Wilson TG \& Corbet EF (2000) Biological complications with dental implants: their prevention, diagnosis and treatment. Clinical Oral Implants Research 11 (Suppl.): 146-155.

11. Andrea Mombelli Andreas Feloutzis Urs Bra gger Niklaus P. Lang Treatment of peri-implantitis by local delivery of tetracycline. Clinical, microbiological and radiological results. Clin Oral Implants Res. 2001 Aug;12(4):287-94.

12. Eugenio Romeo, Marco Ghisolfi, Nicola Murgolo, Matteo resective surgery Chiapasco, Diego Lops, Giorgio Vogel
Therapy of peri-implantitis with A 3-year clinical trial on rough screw-shaped oral implants. Part I: clinical outcome.

13. Ramfjord SP, Nissle RR. The modified widman flap.J Periodontol. 1974 Aug;45(8):601-7.

14. Schou S, Berglundh T, Lang NP Int J Oral Maxillofac Implants. 2004;19 Suppl:140-9.Surgical treatment of peri-implantitis.

15. Zablotsky M. The surgical management of osseous defects associated with endosteal hydroxyapatitecoated and titanium dental implants. Dental Clinics of North America. 1992.

16. Bergendal T, Forsgren L, Kvint S, Lowstedt EThe effect of an airbrasive instrument on soft and hard tissues around osseointegrated implants. A case report. Swed Dent J. 1990;14(5):219-23.

17. Ericsson I, Persson LG, Berglundh T, Edlund T, Lindhe J. The effect of antimicrobial therapy on periimplantitis lesions. An experimental study in the dog Clin Oral Implants Res. 1996 Dec;7(4):320-8.

18. Maintenance of implants: an in vitro study of titanium implant surface modifications subsequent to the application of different prophylaxis procedures $\mathrm{S}$. Matarassol, G. Quaremba2, F. Coraggiol, E. Vaial, C. Cafierol, N. P. Lang3Clinical Oral Implants Research 1996.

19. Mengel R, Buns CE, Mengel C, Flores-de-Jacoby L. An in vitro study of the treatment of implant surfaces with different instruments. Int J Oral Maxillofac Implants. 1998 Jan-Feb; 13(1):91-6.

20. Sicilia A, Noguerol B, Rodríguez ME. Periimplantología. Periodoncia 1994;4:12-26.

21. Mombelli A, Lang NP. The diagnosis and treatment of peri-implantitis. Periodontol 2000. 1998 Jun;17:63-16. Review. 